International Journal of Pure and Applied Mathematics

Volume 90 No. 4 2014, 469-483

ISSN: 1311-8080 (printed version); ISSN: 1314-3395 (on-line version)

url: http://www.ijpam.eu

doi: http://dx.doi.org/10.12732/ijpam.v90i4.9

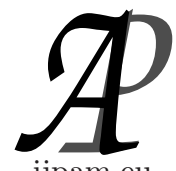

ijpam.eu

\title{
ASYMPTOTIC BEHAVIOUR OF SOLUTIONS OF CERTAIN SECOND ORDER NON-AUTONOMOUS NONLINEAR ORDINARY DIFFERENTIAL EQUATION
}

\author{
J.G. Alaba ${ }^{1 \S}$, B.S. Ogundare ${ }^{2}$ \\ ${ }^{1,2}$ Department of Mathematics \\ Obafemi Awolowo University \\ Ile-Ife, 220005, NIGERIA
}

\begin{abstract}
In this study, we established sufficient criteria for uniform asymptotic stability and boundedness of solutions of certain class of second order nonlinear non autonomous ordinary differential equations using a complete Lyapunov function. Our results extend some existing one in the literature.
\end{abstract}

AMS Subject Classification: 34A34, 34D20, 34D23, 34D99

Key Words: uniform asymptotic stability, boundedness, Lyapunov function, nonlinear non autonomous differential equation

\section{Introduction}

Consider the second order non autonomous ordinary differential equation of the form

$$
\ddot{x}+a(t) f(x, \dot{x}) \dot{x}+b(t) g(x)=p(t ; x, \dot{x})
$$

with an equivalent system

$$
\begin{aligned}
& \dot{x}=y \\
& \dot{y}=-a(t) f(x, y) y-b(t) g(x)+p(t ; x, y)
\end{aligned}
$$

where $f \in C(\mathbb{R} \times \mathbb{R}, \mathbb{R}), g \in C(\mathbb{R}, \mathbb{R}), p \in C([0, \infty) \times \mathbb{R} \times \mathbb{R}, \mathbb{R})$ and $a, b$ are positive functions.

Received: October 18, 2013

(C) 2014 Academic Publications, Ltd. url: www.acadpubl.eu

$\S_{\text {Correspondence author }}$ 
The functions $a, b, f, g$ and $p$ depend only on the arguments displayed explicitly and they are such that existence, uniqueness and continuous dependence on the initial condition are guaranteed. The dots indicate differentiation with respect to the independent variable $t$.

The Lyapunov function or functional approach has been a powerful tool to ascertain the stability/instability, boundedness and periodicity of solutions of ordinary differential equations. Up to today perhaps the most effective method to determine the stability of solutions of nonlinear differential equations is still the Lyapunov's direct (or second) method (see Ezeilo and Ogbu 2010 [11]).

The major advantage of this method is that the stability of solutions can be obtained without any prior knowledge of solutions. However, the construction of these Lyapunov functions remain a general problem due to lack of unique way for its construction. Many methods have been proposed in the literatures (see for instance [9], [10], [22], [23], [35], [25], [26], [12], [13], [14], [15], [16], [37], [36], [22], [23], [24], [19], [45], [29], [43], [44]).

Over the years, numerous authors have dealt with problems of second order nonlinear ordinary differential equations and they obtained many interesting results on qualitative properties of solutions such as stability, boundedness, periodicity and convergence (see for examples [4], [2], [3], [5], [6], [7], [17], [18], [24], [25], [31], [32], [39], [40], [41], [42], [28], [30], [38], [34], [34], [27]) and the references cited therein.

In many of these works, the authors made use of Lyapunov's direct method by constructing Lyapunov functions and obtained criteria which guarantee some qualitative behaviours of their solutions.

Many special cases of (1.1) exist in the literature especially the autonomous problem (i.e, when $a(t)=b(t)=1$ or constant independent of $t$ cf.[1], [18], [33] and references contained in them).

In particular, Cautareli[8] investigated stability of solutions of certain class of (1.1) using Lyapunov's direct method and constructed several different Lyapunov functions. In 2007, Tunc.C and Tun.E[40] discussed the global asymptotic stability of the trivial solutions of non autonomous system with application to second order equation by means of Lyapunov's direct method. Ogundare and Okecha[31] obtained criteria for global asymptotic stability, boundedness and the existence of periodic solutions with the aid of Lyappunov's direct method.

The motivation for this study basically is the works of Cautareli[8], Ogundare and Okecha[31], E. Tunc and C. Tunc[40]. Our main objectives are to revise, extend and improve their obtained results with less restrictive conditions.

We shall give with the use of a suitable Lyapunov function, sufficient con- 
ditions on the nonlinear terms $f$ and $g$ as well as on the functions $a, b$ and $p$ that will guarantee the existence of a unique solution which is globally asymptotically stable and bounded together with its derivative on the real line.

\section{The Main Results}

\subsection{Basic Assumptions}

The following are the basic assumptions used to formulate our main results for this article.

Let $a, b, f, g$ and $p$ be continuous functions on interval $I=[0, a b], a$ and $b$ are positive functions i.e, $a, b>0$ with $0<a_{0}<a \leq a(t) \leq a_{1}$ and $0<b_{0}<b \leq b(t) \leq b_{1}$ for all $t \in I$; in addition, $a$ and $b$ are differentiable with $a(t)$ and $b(t)$ being decreasing functions i.e, $\dot{a} \leq 0$ and $\dot{b} \leq 0$.

(i) $|f(x, y)| \leq \beta \in I$,

(ii) $G_{0}=\frac{g(x)-g(0)}{x} \leq \alpha \in I$, with $x \neq 0, g(0)=0$ and $\alpha, \beta>0$.

(iii) $\beta(\delta+1)(\dot{a} b-a \dot{b})>\dot{a} a^{2} \alpha^{2}$ for all $\alpha, \beta, \delta$ all positive.

(iv) $p(t ; x, y)=p(t)$ and $|p(t)| \leq Z$ for all $t \leq 0$, where $Z$ is a constant.

(v) $|p(t ; x, y)| \leq(|x|+|y|) \phi(t)$, where $\phi(t)$ is a non-negative and continuous function of $t$ and satisfies $\int_{0}^{t} \phi(s) d s \leq Z<\infty, Z>0$ is a constant.

The main results with respect to (1.1) are given below:

Theorem 2.1. Suppose the conditions (i)- (iii) are satisfied with $p(t ; x, \dot{x}) \equiv$ 0 , then the trivial solution of (1.2) is globally asymptotically stable.

Theorem 2.2. Suppose that the conditions of Theorem 2.1 hold and in addition:

(iv) $p(t ; x, y)=p(t)$ and $|p(t)| \leq Z$ for all $t \leq 0$, where $Z$ is a constant.

Then there exists $\sigma(0<\sigma<\infty)$ depending only on $\alpha, \beta$ and $\delta$ such that every solution of system (1.2) satisfies

$$
x^{2}(t)+\dot{x}^{2}(t) \leq e^{-\sigma t}\left\{Q_{1}+Q_{2} \int_{t_{0}}^{t}|p(\tau)| e^{\left(\frac{\sigma \tau}{2}\right)} d \tau\right\}^{2}
$$

for all $t \geq t_{0}$, where the constant $Q_{1}>0$ depends on $\alpha, \beta, \delta, t_{0}, x\left(t_{0}\right), \dot{x}(t)$ and the constant $Q_{2}>0$ depends on $\alpha, \beta$ and $\delta$ only. 
Theorem 2.3. Suppose the conditions of Theorem 2.2 with condition (iv) replaced with

$(\mathrm{v})|p(t ; x, y)| \leq(|x|+|y|) \phi(t)$, where $\phi(t)$ is a non-negative and continuous function of $t$ and satisfies $\int_{0}^{t} \phi(s) d s \leq Z<\infty, Z>0$ is a constant.

Then there exists a constant $\lambda_{0}$ which depends on $Z, \lambda_{1}, \lambda_{2}$ and $t_{0}$ such that every solution $x(t)$ of system (1.2) satisfies $|x(t)| \leq \lambda_{0}, \quad|\dot{x}(t)| \leq \lambda_{0}$ for sufficiently large $t$.

\section{Preliminary Results}

The main tool to use in proving our main results is the scalar function $V(t ; x, y)$ defined below

$$
V(t ; x, y)=\frac{1}{2 a \alpha}\left\{(a \alpha)^{2} x^{2}+(\delta+1) b \beta x^{2}+\delta y^{2}+y^{2}+2 a \alpha x y\right\}
$$

where $a=a(t), b=b(t)$ and $\forall \alpha, \beta, \delta>0$.

We need to first established that (3.1.1) is indeed a Lyapunov function for the system (1.2) and we shall state the followings Lemmas and proofs.

Lemma 3.1. Subject to the assumptions of Theorem 2.1, there exist positive constants

$$
\lambda_{i}=\lambda_{i}(a, b, \alpha, \beta, \delta), \quad i=1,2
$$

such that

$$
\lambda_{1}\left(x^{2}+y^{2}\right) \leq V(t ; x, y) \leq \lambda_{2}\left(x^{2}+y^{2}\right)
$$

Proof. Clearly, $V(t ; 0,0)=0$.

Re-write equation (3.1) gives

$$
\begin{gathered}
V(t ; x, y)=\frac{1}{2 a \alpha}\left\{(a \alpha x+y)^{2}+(\delta+1) b \beta x^{2}+\delta y^{2}\right\} \\
=\frac{1}{2 a \alpha}\left\{(a \alpha x+y)^{2}+[(\delta+1) b \beta] x^{2}+\delta y^{2}\right\} \\
\geq \frac{1}{2 a \alpha}\left\{[(\delta+1) b \beta] x^{2}+\delta y^{2}\right\} \\
V(t ; x, y) \geq \lambda_{1}\left(x^{2}+y^{2}\right)
\end{gathered}
$$

with

$$
\lambda_{1}=\lambda_{0} \cdot \min \left\{(\delta+1) b_{0} \beta, \delta\right\} \quad \text { and } \quad \lambda_{0}=\frac{1}{2 a \alpha}
$$


Applying the inequality $|x y| \leq \frac{1}{2}\left|x^{2}+y^{2}\right|$ on (3.1.1) gives

$$
\begin{gathered}
V(t ; x, y) \leq \frac{1}{2 a \alpha}\left\{\left[(a \alpha)^{2}+(\delta+1) b \beta\right] x^{2}+[\delta+1] y^{2}+a \alpha x^{2}+a \alpha y^{2}\right\} \\
V(t ; x, y) \leq \frac{1}{2 a \alpha}\left\{\left[(a \alpha)^{2}+(\delta+1) b \beta+a \alpha\right] x^{2}+[\delta+1+a \alpha] y^{2}\right\} \\
V(t ; x, y) \leq \lambda_{2}\left(x^{2}+y^{2}\right)
\end{gathered}
$$

with

$$
\lambda_{2}=\lambda_{0} \cdot \max \left\{\left(a_{1} \alpha\right)^{2}+(\delta+1) b_{1} \beta+a_{1} \alpha, \delta+1+a_{1} \alpha\right\}
$$

From equations (3.1.5) and (3.1.8), we have

$$
\lambda_{1}\left(x^{2}+y^{2}\right) \leq V(t ; x, y) \leq \lambda_{2}\left(x^{2}+y^{2}\right)
$$

This completes the proof of Lemma 3.1

Lemma 3.2. Subject to the assumptions of Theorem 2.1, there exist positive constants $\lambda_{j}=\lambda_{j}(a, b, \alpha, \beta, \delta)$ with $j=3,4$ such that for any solution $(x, y)$ of system (1.2)

$\left.\frac{d V}{d t}\right|_{(1.2)}=\left.\dot{V}(t ; x, y)\right|_{(1.2)}=\left.\frac{d V(t ; x, y)}{d t}\right|_{(1.2)} \leq-\lambda_{3}\left(x^{2}+y^{2}\right)+\lambda_{4}(|x|+|y|)|p(t ; x, y)|$.

Proof. The time derivative of (3.1.1) along the system (1.2) is defined below

$$
\left.\frac{d V(t ; x, y)}{d t}\right|_{(1.2)}=\left.\dot{V}\right|_{(1.2)}=\frac{\partial V}{\partial t}+\frac{\partial V}{\partial x} \dot{x}+\frac{\partial V}{\partial y} \dot{y}
$$

where

$$
\begin{aligned}
\frac{\partial V}{\partial t}=\frac{1}{4 a^{2} \alpha^{2}}\{2 a \alpha & \left(\left[2 \dot{a} a \alpha^{2}+\dot{b} \beta(\delta+1)\right] x^{2}+2 \dot{a} \alpha x y\right)-2 \dot{a} \alpha\left(\left[(a \alpha)^{2}\right.\right. \\
+ & \left.\left.(\delta+1) b \beta] x^{2}+(\delta+1) y^{2}+2 a \alpha x y\right)\right\} \\
= & \frac{1}{4 a^{2} \alpha^{2}}\left\{\left[2 \dot{a} a^{2} \alpha^{3}+2(\delta+1) \alpha \beta(a \dot{b}-\dot{a} b)\right] x^{2}-[2 \dot{a} \alpha(\delta+1)] y^{2}\right\} .
\end{aligned}
$$

Further simplification gives

$$
\begin{gathered}
\frac{\partial V}{\partial t}=\frac{1}{2 a \alpha}\left\{\left[\dot{a} a \alpha^{2}+\frac{(\delta+1)}{a} \beta(a \dot{b}-\dot{a} b)\right] x^{2}-\left[\frac{\dot{a}}{a}(\delta+1)\right] y^{2}\right\} \\
\frac{\partial V}{\partial x} \dot{x}=\frac{1}{a \alpha}\left\{\left[(a \alpha)^{2}+(\delta+1) b \beta\right] x y+a \alpha y^{2}\right\}
\end{gathered}
$$


and

$$
\begin{array}{r}
\frac{\partial V}{\partial y} \dot{y}=\frac{1}{a \alpha}\left\{-(a \alpha)^{2} x y-a(\delta+1) \alpha y^{2}-a b \alpha \beta x^{2}-b \beta(\delta+1) x y\right. \\
+p(t ; x, y) a \alpha x+p(t ; x, y)(\delta+1) y\} .
\end{array}
$$

Putting 3.2.1a, 3.2.1b and 3.2.1c in 3.2.1 yields

$$
\begin{aligned}
\left.\frac{d V(t ; x, y)}{d t}\right|_{(1.2)}= & -\frac{1}{a \alpha}\left\{\left(a b \alpha \beta-\left[\frac{\dot{a} a \alpha^{2}}{2}+\frac{(\delta+1)}{2 a} \beta(a \dot{b}-\dot{a} b)\right]\right) x^{2}\right. \\
& +\left(\frac{\dot{a}}{2 a}(\delta+1)+a \alpha(\delta+1)\right) y^{2} \\
& +p(t ; x, y)[a \alpha x+(\delta+1) y]\}
\end{aligned}
$$

Further simplification of (3.2.2) yields

$$
\begin{aligned}
\frac{d V}{d t}= & -\frac{1}{2 a \alpha}\left\{\left(\frac{2 a^{2} b \alpha \beta+\beta(\delta+1)(\dot{a} b-a \dot{b})-\dot{a} a^{2} \alpha^{2}}{a}\right) x^{2}\right. \\
& +\left(\frac{\dot{a}(\delta+1)+2 a^{2} \alpha(\delta+1)}{a}\right) y^{2} \\
& +p(t ; x, y)(a \alpha x+(\delta+1) y)\} \\
& \dot{V}(t ; x, y) \leq-\lambda_{3}\left(x^{2}+y^{2}\right)+\lambda_{*}(|x|+|y|) p(t ; x, y)
\end{aligned}
$$

with $\lambda_{0}=\frac{1}{2 a \alpha}, \lambda_{*}=\{a \alpha,(\delta+1)\}$ and

$$
\lambda_{3}=\lambda_{0} \cdot \max \left\{\frac{2 a^{2} b \alpha \beta+\beta(\delta+1)(\dot{a} b-a \dot{b})-\dot{a} a^{2} \alpha^{2}}{a}, \frac{\dot{a}(\delta+1)+2 a^{2} \alpha(\delta+1)}{a}\right\}
$$

Inequality (3.2.4) can also be simplified and given as

$$
\dot{V}(t ; x, y) \leq-\lambda_{3}\left(x^{2}+y^{2}\right)+\lambda_{4}\left(x^{2}+y^{2}\right)^{\frac{1}{2}}|p(t ; x, y)|
$$

with $\lambda_{4}=\sqrt{2} \lambda_{*}$

This completes the proof of Lemma 3.2

Remarks 3.1. When $p(t ; x, y) \equiv 0,(3.2 .5)$ becomes

$$
\frac{d V}{d t}=\dot{V}(t ; x, y) \leq-\lambda_{3}\left(x^{2}+y^{2}\right)
$$




\section{Proof of the Main Results}

Now, we shall give the proofs of our main results stated in Section 2 as follows:

Proof of Theorem 2.1. From Lemma (3.1) and (3.2) with $p(t ; x, \dot{x}) \equiv 0$, it had been established that the function $V(t ; x, y)$ of $(3.1 .1)$ is a Lyapunov function, for the system (1.2).

Hence, the trivial solution of system (1.2) is globally asymptotically stable (g.a.s), that is, every solution $x(t), \dot{x}(t)$ of the system (1.2) satisfies $x^{2}(t)+$ $\dot{x}^{2}(t) \rightarrow 0$ as $t \rightarrow \infty$.

Remark 4.1. For a homogeneous equation (when $p(t ; x, y) \equiv 0$ ), the proofs of Lemmas 3.1 and 3.2 shows that the equation (1.1) has a solution that is asymptotically stable that is, equation (3.2.6) In fact uniformly asymptotically stable (u.a.s).

Proof of Theorem 2.2. From (3.2.5) replacing $p(t ; x, \dot{x})$ with $p(t)$,

$$
\dot{V}(t ; x, y) \leq-\lambda_{3}\left(x^{2}+y^{2}\right)+\lambda_{4}\left(x^{2}+y^{2}\right)^{\frac{1}{2}} p(t)
$$

From (3.1.5), we have

$$
\left(x^{2}+y^{2}\right)^{\frac{1}{2}} \leq\left(\frac{V}{\lambda_{1}}\right)^{\frac{1}{2}}
$$

Also from (3.1.5), we have

$$
\lambda_{3}\left(x^{2}+y^{2}\right) \leq \lambda_{3} \cdot \frac{V}{\lambda_{1}}
$$

using equations (4.1.2) and (4.1.3), thus (4.1.1) becomes

$$
\frac{d V}{d t} \leq-\lambda_{6} V+\lambda_{5} V^{\frac{1}{2}}|p(t)|
$$

where $\lambda_{6}=\frac{\lambda_{3}}{\lambda_{1}}$ and $\lambda_{5}=\frac{\lambda_{4}}{\lambda_{1}^{\frac{1}{2}}}$ equation (4.1.4) can also be expressed as

$$
\frac{d V}{d t} \leq-2 \lambda_{7} V+\lambda_{5} V^{\frac{1}{2}}|p(t)|
$$

with $\lambda_{7}=\frac{\lambda_{6}}{2}$

Therefore,

$$
\begin{gathered}
\dot{V}+\lambda_{7} V \leq-\lambda_{7} V+\lambda_{5} V^{\frac{1}{2}}|p(t)| \\
\dot{V}+\lambda_{7} V \leq \lambda_{5} V^{\frac{1}{2}}\left\{|p(t)|-\lambda_{8} V^{\frac{1}{2}}\right\}
\end{gathered}
$$


where $\lambda_{8}=\frac{\lambda_{7}}{\lambda_{5}}$

Thus (4.1.7) becomes

$$
\dot{V}+\lambda_{7} V \leq \lambda_{5} V^{\frac{1}{2}} V^{*}
$$

where

$$
V^{*}=|p(t)|-\lambda_{8} V^{\frac{1}{2}}
$$

when $|p(t)| \leq \lambda_{8} V^{\frac{1}{2}}$, then (4.1.9) becomes

$$
V^{*} \leq 0
$$

and when $|p(t)| \geq \lambda_{8} V^{\frac{1}{2}}$, then (4.1.9) becomes

$$
V^{*} \geq 0
$$

Substituting (4.1.10) into (4.1.7), we have

$$
\dot{V}+\lambda_{7} V \leq \lambda_{9} V^{\frac{1}{2}}|p(t)|
$$

where $\lambda_{9}=\frac{\lambda_{5}}{\lambda_{8}}$

This implies that

$$
\dot{V} V^{-\frac{1}{2}}+\lambda_{7} V^{\frac{1}{2}} \leq \lambda_{9}|p(t)|
$$

Multiplying both sides of (4.1.13) by $e^{\frac{\lambda_{7} t}{2}}$, we have

$$
e^{\frac{\lambda_{7} t}{2}}\left\{\dot{V} V^{-\frac{1}{2}}+\lambda_{7} V^{\frac{1}{2}}\right\} \leq e^{\frac{\lambda_{7} t}{2}} \lambda_{9}|p(t)|
$$

that is,

$$
2 \frac{d}{d t}\left\{V^{\frac{1}{2}} e^{\left(\frac{\lambda_{7} t}{2}\right)}\right\} \leq e^{\frac{\lambda_{7} t}{2}} \lambda_{9}|p(t)|
$$

integrating both sides of (4.1.15) from $t_{0}$ to $t$, gives

$$
\left\{V^{\frac{1}{2}} e^{\frac{\lambda_{7} \xi}{2}}\right\}_{t_{0}}^{t} \leq \int_{t_{0}}^{t} \frac{1}{2} e^{\frac{\lambda_{7} \tau}{2}} \lambda_{9}|p(\tau)| d \tau
$$

which implies that

$$
\left\{V^{\frac{1}{2}}(t)\right\} e^{\left(\frac{\lambda_{7} t}{2}\right)} \leq V^{\frac{1}{2}}\left(t_{0}\right) e^{\frac{\lambda_{7} t_{0}}{2}}+\frac{\lambda_{9}}{2} \int_{t_{0}}^{t}|p(\tau)| e^{\frac{\lambda_{7} \tau}{2}} d \tau
$$

or

$$
V^{\frac{1}{2}}(t) \leq e^{-\left(\frac{\lambda_{7} t}{2}\right)}\left\{V^{\frac{1}{2}}\left(t_{0}\right) e^{\frac{\lambda_{7} t_{0}}{2}}+\frac{\lambda_{9}}{2} \int_{t_{0}}^{t}|p(\tau)| e^{\frac{\lambda_{7} \tau}{2}} d \tau\right\}
$$


using (3.1.5) and (4.1.17), we have

$$
\lambda_{1}\left(x^{2}(t)+\dot{x}^{2}(t)\right) \leq e^{-\left(\lambda_{7} t\right)}\left\{\lambda_{2}\left(x(t)^{2}+\dot{x}^{2}(t)\right)^{\frac{1}{2}} e^{\frac{\lambda_{7} t_{0}}{2}}+\frac{\lambda_{9}}{2} \int_{t_{0}}^{t}|p(\tau)| e^{\frac{\lambda_{7} \tau}{2}} d \tau\right\}^{2}
$$

for all $t \geq 0$.

Thus

$$
\begin{gathered}
x^{2}(t)+\dot{x}^{2}(t) \leq \frac{1}{\lambda_{1}}\left\{e^{-\lambda_{7} t}\left\{\lambda_{2}\left(x^{2}(t)+\dot{x}^{2}(t)\right)^{\frac{1}{2}} e^{\frac{\lambda_{7} t_{0}}{2}}+\frac{\lambda_{9}}{2} \int_{t_{0}}^{t}|p(\tau)| e^{\frac{\lambda_{7} \tau}{2}} d \tau\right\}^{2}\right\} \\
\leq e^{-\lambda_{7} t}\left\{Q_{1}+Q_{2} \int_{t_{0}}^{t}|p(\tau)| e^{\frac{\lambda_{7} \tau}{2}} d \tau\right\}^{2}
\end{gathered}
$$

where $Q_{1}=\lambda_{2}\left(x(t)^{2}+\dot{x}^{2}(t)\right)^{\frac{1}{2}} e^{\frac{\lambda_{7} t_{0}}{2}}$ and $Q_{2}=\frac{\lambda_{9}}{2}$

By putting $\lambda_{7}=\sigma$ in the inequality(4.1.20) and gives

$$
x^{2}(t)+\dot{x}^{2}(t) \leq e^{-\sigma t}\left\{Q_{1}+Q_{2} \int_{t_{0}}^{t}|p(\tau)| e^{\left(\frac{\sigma \tau}{2}\right)} d \tau\right\}^{2}
$$

Hence, this completes the proof.

Remark 4.2. From the proof of the Theorem 2.2, the following can be pointed out as the direct consequence of the Theorem 2.2. If $p(t, x, y) \equiv 0$, then the trivial solution of (1.2) is uniformly asymptotically stable.

Remark 4.3. If $p(t ; x, y) \equiv 0$, then (4.1.21) reduces to

$$
x^{2}(t)+\dot{x}^{2}(t) \leq e^{-\sigma t} Q_{1},
$$

as $t \rightarrow \infty, x^{2}(t)+\dot{x}^{2}(t) \rightarrow 0$ which implies that the trivial solution of the system (1.2) or better say the equation (1.1) is globally asymptotically stable.

Proof of Theorem 2.3. Indeed from the inequality (4.2.4), we have

$$
\dot{V} \leq \lambda_{*}(|x|+|y|)^{2} \phi(t)
$$

By using the Schwartz inequalities on (4.1.22) yields

$$
\dot{V} \leq \lambda_{10}\left(x^{2}+y^{2}\right) \phi(t)
$$

where $\lambda_{10}=2 \lambda_{*}$ 
From the inequalities (3.1.5) and (4.1.17), we have

$$
\dot{V} \leq \lambda_{10} V \phi(t)
$$

integrating equation (4.1.23) from 0 to $t_{0}$, we obtain

$$
V(t)-V(0) \leq \lambda_{11} \int_{t_{0}}^{t} V(s) \phi(s) d s
$$

where $\lambda_{11}=\frac{\lambda_{10}}{\lambda_{1}}=\frac{3 \lambda_{*}}{\lambda_{1}}$

The inequality (4.1.25) now becomes

$$
V(t) \leq V(0)+\lambda_{11} \int_{t_{0}}^{t} V(s) \phi(s) d s
$$

By Gronwall-Bellman inequality (4.1.26) yields

$$
V(t) \leq V(0) \exp \left(\lambda_{11} \int_{t_{0}}^{t} \phi(s) d s\right)
$$

This completes the proof.

\section{Application}

As an application to our obtained result we consider the example below

\subsection{Example}

Consider second order non autonomous nonlinear differential equation

$$
x^{\prime \prime}+\frac{2 t}{1+t^{2}} x^{\prime}+6[2-\exp (-t)] x^{5}=\frac{\exp (t)}{1+\exp (2 t)+x^{2}+x^{\prime 2}}
$$

We state (5.1) as the system form,

$$
\begin{aligned}
& x^{\prime}=y, \\
& y^{\prime}=-\frac{2 t}{1+t^{2}} y-6[2-\exp (-t)] x^{5}+\frac{\exp (t)}{1+\exp (2 t)+x^{2}+x^{\prime 2}} .
\end{aligned}
$$

Comparing (5.1) with (1.1), it is easily seen that

$$
a(t)=\frac{2 t}{1+t^{2}}, t \leq 0,
$$




$$
\begin{gathered}
0 \leq \frac{2 t}{1+t^{2}} \leq 1, \\
a_{0}=0, a_{1}=1 . \\
b(t)=6[2-\exp (-t)], t \leq 0, \\
6 \leq 6[2-\exp (-t)] \leq 12, \\
b_{0}=6, b_{1}=12 . \\
\lambda_{0}=\frac{1}{\alpha}, \lambda_{3}=12 \beta .
\end{gathered}
$$

The corresponding Lyapunov function to the system (5.2) is given

$$
V=\frac{1}{2 \alpha}\left\{12 \beta(\delta+1) x^{2}+\delta y^{2}+(\alpha x+y)^{2}\right\}>0
$$

where $\alpha, \beta$, and $\delta$ are positive constants and whose derivative is given

$$
\frac{d V}{d t}=\dot{V}(t ; x, y) \leq-12 \beta\left(x^{2}+y^{2}\right)
$$

where $\beta>0$.

All conditions stated in Theorem 2.1 are satisfied therefore the zero solution of system (5.2) is globally asymptotic stable.

We have for $p \equiv 0$ that the solutions of (5.1) are globally asymptotic stable.

\section{References}

[1] Atkinson, F.V. On second order nonlinear oscillations, Pacific J. Math. Vol.5, (1955), pp.643-647.

[2] Baker, J.W. Stability Properties of a Second Order Damped and Forced Nonlinear Differential Equation, SIAM J.Appl. Math. Vol.27, No.1, (1974), pp.159-166

[3] Bihari, I. On periodic solutions of certain second order ordinary differential equations with periodic coefficients, Acta. Math. Acad. Sci. Hungar. Vol.12, (1961) pp.11-16. doi no:10.1007/BF02066674.

[4] Burton, T.A. and Shunian, Z. Unified Boundedness, Periodicity and Stability in Ordinary and Functional Differential Equation, Ann. Mat. Pura Appl.. Vol. 145, (1986), pp.129-158. doi no:10.1007/BF01790540. 
[5] Burton, T.A. Lyapunov functions and boundedness, J. Math. Anal. Appl.Vol.58, (1977), pp. 88-97 doi no: 10.1016/0022-247X(77)90230-X.

[6] Cartwright, M.L. On stability of solutions of certain differential equations of the fourth-order. Quart. J. Mech. and Appl. Math., Vol.9, (1956), pp.185-194,doi no: 10.1093/qjmam/9.2.185.

[7] Cartwright, M.L and Littlewood, J.E. On nonlinear differential equations of the second order, Annals of Math., Vol.48, (1947) pp.472-494, $\operatorname{MR0021190(9,35c)~}$

[8] Cautareli, G. On the stability of the origin of a non autonomous Lienard equation, Boll. Uni. Mat. Ital. A Vol.7 No.10, (1996),pp.563-573.

[9] Chin, P.S.M. Stability of nonlinear systems via the intrinsic method. Int. J. Control, Vol.48, No.4, (1988), pp.933 - 943, MR0964780.

[10] Chin, P.S.M. Extension to the intrinsic method and its application to stability problems. Int. J. Control, Vol.48, (1988), pp.2139-2146, MR0973781(89i:34073).

[11] Ezeilo, J.O.C and Ogbu, H.M. Costruction of Lyapunov-Type Functions of Some Third Order Nonlinear Ordinary Differential Equations by the Method of Integration. J of Sci. Teacher Association of Nigeria Vol.45, (2010), pp.1-2.

[12] Ezeilo, J.O.C. On boundedness of solutions of certain differential equations of third order. Proceedings of Cambridge Philosophical Society Vol.9,(1959), pp.74-114.

[13] Ezeilo, J.O.C. Instability of solutions of certain differential equations of third order. Proceedings of Cambridge Philosophical Society Vol.56,(1960) pp.81-387.

[14] Ezeilo, J.O.C. A boundedness theorem for some third differential equations. Journal of London Mathematical Society Vol.36, (1967) pp.439-444.

[15] Gurel, O and Lapidus, L. A Guilde to the Generation of Liapunov functions, Ind. Eng. Chem., Vol.61, No.3, (1969), pp.30-41

[16] Hahn, W. Theory and Application of Lyapunov's Direct Method, PrenticeHall, Inc, New Jersey,(1963), doi no: 10.1063/1.3051152. 
[17] Halanay, A. A qualitative theory of differential equations, Lyapunov stability of oscilating system with deviating arguments. Ed. Accad. Rep. Prop. Romania Buchmest Translation Academic Press, New York (1962).

[18] Hatvani, L. On the stability of the zero solution of nonlinear second order differential equations, Acta Sci. Math. (Szeged) Vol. 32,(1971) pp.1-9.

[19] Hatvani, L. On the strong stability by Lyapunov's direct method, Acta. Sci. Math. (Szeged), Vol.37,(1972) pp.241-251.

[20] Hatvani, L. On stability properties of solutions of second order differential equations,Electron. j. Qual. Theory Differ. Equ., Szeged Vol.11, No.6, (2000),pp.

[21] Hongxing, Y and Weiye, M. On the Stability of Solutions of Certain Nonlinear Third-Order Delay Differential Equations. Int. J. of Nonlinear Sci.China. Vol.6, No.3,(2008) pp.230-237, .

[22] Krasovskii, N. N. Stability of motion, stanford University press, stanford,(1963) MR0147744 (26 A2712), doi no:10.1016/0021-8928(63)90181-3.

[23] Lakshmikantam, V, Leela, S, Martynyuk, A.A. Stability analysis of nonlinear systems Monographs and Textbooks in Pure and Applied Mathematics, 125. Marcel Dekker, Inc., New York (1989).

[24] Leighton, W . On Construction of Lyapunov function for certain autonomous differential equations, Contrib. Diff. Equ.Vol.2,(1963) pp.367383.

[25] Lassalle, J.P and Lefschetz, S. Stability by Lyapunov's direct method with Applications, Academic press, Interscience, New York, (1961).

[26] Lassalle, J.P and Hale, J. K. Differential Equations: Linearity vs Nonlinearity, SIAM Review USA, Vol.5, No.3 (1963) pp. 249-272 .

[27] Lim, Y.S and Kazda, L.F A study of second order nonlinear systems, Journal of Math. Anal. and Appl.USA Vol.8,(1964) pp.423-444,doi no:10.1016/0022-247X(64)90052-6.

[28] Loud, W.S. Boundedness and Convergence of solutions of $\ddot{x}+c \dot{x}+g(x)=$ $\exp (t)$. Duke Math. J. Vol.24,(1957) pp.63-72, MR0085415 (19,36c), doi no:10.1215/S0012-7094-57-02412-2 
[29] Lyapunov, A.M. The general problem of stability of motion (in Russia) [Translated and Reproduced in Ann.Math. study Vol.17(1947) Princeton, New Jersey (1892), doi no:10.1080/00207179208934253.

[30] Napoles, J.E and Valdes A note on the asymptotic stability in the whole of non autonomous systems, Rev. Colombiana Mat.Vol.33,(1999) pp.1-8.

[31] Ogundare, B.S and Okecha, G. E. Boundedness, periodicity and stability of solutions to $\ddot{x}+a(t) g(\dot{x})+b(t) h(x)=p(t ; x, \dot{x})$, Math. Sci. Res.J., Vol.11 No.5, (2007), pp.432-443.

[32] Ogundare, B. S. Ngcibi, S and Murali, V. Boundedness and Stability Properties of Solutions to certain second order Differential Equation. Allahabad, India Vol.5, No. 2,(2010) pp 79-92.

[33] Pucci, P and Serrin, J. Precise damping conditions for global asymptotic stability for nonlinear second order systems, Acta. Math. Vol.170,(1993), pp.275-307, doi no:10.1007/BF02392788.

[34] Pucci .P and Serrin, J. Precise damping conditions for global asymptotic stability for nonlinear second order systems, J. Differential Equations Vol.1l No.113,(1994) pp.505-534, doi no: 10.1006/jdeq.1994. 1134.

[35] Reissig, G. Sansone, R. Conti Nonlinear differential equations of higher order, Noordhoff International Publishing. A division of A.W. Sijthoff International Publishing company ISBN: 9001752705, B.V., Leyden, Netherland, (1974).

[36] Rouche, N., Habets, P and Laloy, M. Stability Theory by Liapunov's Second Method, Spring-Verlag, Heidelberg, Berlin, (1977).

[37] Schultz, D.G and Gibson, J.E. The Variable Gradient Method for Generating Lyapunov Functions, AIEE Trans. Part ll, Vol.81, (1962),pp.203-209, doi no: 10.1109/TAI.1962.6371818.

[38] Sugie, J. and Amano, Y. Global asymptotic stability of non autonomous systems of Lienard type, J. Math. Anal. Appl. Vol.289 No.2, pp.673-690.

[39] Tejumola, H. O. Boundedness Criteria for solutions of some second-order differential equations, Atti. Accad. Naz. Lincei Rend. Cl. Sci. Fis. Math. Natur., Vol.50, (1971),pp.432-437. 
[40] Tunc, C and Tunc, E. On the asymptotic behaviour of solutions of certain second order differential equations.J.Franklin Inst.Vol.344,(2007), pp.391398.

[41] Tunc. C. On the Boundedness of solutions of a non-autonomous differential equation of second order. Sarajevo Jour. of Math. Vol.17,(2011) pp.19-29

[42] Wong, J. S. W and Burton, T. A. Some Properties of Solutions of second order nonlinear non autonomous differential equation, Monnatshefte Fur Mathematik, Vol.69, (1965) pp.368-374

[43] Yoshizawa, T. Stability Theory by Lyapunov's Second Method The Mathematical Society of Japan, Tokyo, (1966).

[44] Yoshizawa, T. Stability theory and existence of periodic solutions and almost periodics, Spring-Verlag, New York, Heidelberg, Berlin,(1975).

[45] Zubov. The methods of Lyapunov and their applications, Noordhoff, Groning,(1964). 
